\title{
MANAGING PULMONARY TUBERCULOSIS IN A PATIENT WITH POST OP OROPHARYNGEAL CANCER - CHALLENGES FOR FAMILY PHYSICIAN
}

\author{
Mohd Shaiful Ehsan Shalihin ${ }^{1 *}$, Zulkifli Harun² \\ ${ }^{1}$ Department of Family Medicine, Kulliyyah of Medicine, International Islamic University of Malaysia, Jalan Sultan \\ Ahmad Shah, 25200 Kuantan, Pahang, Malaysia. \\ ${ }^{2}$ Klinik Kesihatan Rompin, Jalan Rompin - Pekan, 26800 Rompin, Pahang, Malaysia.
}

Corresponding author: Dr Mohd Shaiful Ehsan Bin Shalihin

Email: shaifulehsan@iium.edu.my

\begin{abstract}
Oropharyngeal carcinoma is uncommon cancer compared to other malignancies. It accounts for less than $3 \%$ worldwide cancer and classified under the head and neck carcinoma. Patients with locally advanced oropharyngeal malignancy are usually treated with surgery with combination of radiotherapy and chemotherapy. This later required regular follow up especially on supportive care and adaptation to swallowing. Difficulty in swallowing provided burden to the patient and may lead to underlying malnutrition and risk of infection including tuberculosis. This will end up with poor compliance to oral treatment and risk of resistance. Therefore, this case study is illustrating the challenging role of family medicine specialists in managing a case of sputum positive pulmonary tuberculosis with underlying swallowing difficulty from glossectomy and radiotherapy on top of defaulted tongue carcinoma supportive care follow up.
\end{abstract}

Keywords: Oropharyngeal carcinoma, Pulmonary tuberculosis, Swallowing

\section{INTRODUCTION}

Oropharyngeal cancer is ranked the tenth most common cancer worldwide with estimated 700,000 new cases per year causing more than 300,000 deaths. ${ }^{1}$ Oral cancers include cancer of the lips, oral cavity, tongue, nasopharynx and pharynx. One aspect of this cancer which is closely related with primary care is the higher probability for the patient to be complicated with pulmonary tuberculosis due to inadequate nutrition secondary to poor oral intake and oropharyngeal destruction by the cancer. ${ }^{2,3}$ Poor nutrition leads to malnutrition and micronutrients deficiencies, major risk factors for immunodeficiency. This increases the patient's susceptibility to infection, especially tuberculosis with 2.9-fold increased hazard risk. ${ }^{2,3}$

Pulmonary tuberculosis is an infection caused by Mycobacterium tuberculosis which majorly affects the lung. ${ }^{4,5}$ Anorexia and weight loss are common features of advanced disease and may be the only presenting features in some patients. However, the symptoms may be less clearly directed to tuberculosis especially in those with underlying poor oral intake or swallowing disorder that might present with similar symptoms. ${ }^{6-9}$ Late diagnosis would further deteriorate the patient's quality of life and underlying comorbid.

For the past decades, we are focusing on treating pulmonary tuberculosis rather than multiplying our efforts in detecting new cases.
Therefore, primary care providers should be more vigilant in screening new cases by identifying high risk group patients. This can be done by examining the patient's sputum for acid fast bacilli as part of investigation that can easily be done at primary care level for those who are symptomatic. ${ }^{6-9}$ Chest $\mathrm{x}$-ray meanwhile can be performed for asymptomatic high risk groups as part of screening. ${ }^{10-12}$

There are now over 30 million cancer survivors worldwide. This number is expected to increase dramatically due to improvements in cancer screening and longer life expectancy following definitive cancer treatment. ${ }^{13,14}$ Patients with underlying malignancy even already being treated by the tertiary level should be under regular follow up at least yearly. ${ }^{15,16}$ This is because risk of recurrence and late complications is always present and may be poorly detected by the patient themselves. Malignancy and malnutrition are established risk factors for tuberculosis infection. ${ }^{6}$ Treatment for tuberculosis in this subgroup would definitely be difficult and inculcate challenges to both the patient and doctor due to underlying defects by the cancer or complications from previous treatment. ${ }^{8-10,17}$ Among the challenges includes difficulty in consuming the medications, adjustment of the dose, non-compliance and risk of prolonged treatment and resistance. 


\section{METHODS}

This case study aims to illustrate the challenging role of family medicine specialists in managing a case of sputum positive pulmonary tuberculosis with underlying swallowing difficulty from glossectomy and radiotherapy of tongue carcinoma.

\section{RESULTS}

We reported a case of a 50-year-old woman with underlying tongue carcinoma, diagnosed 20 years ago when she presented with tongue mass and speech difficulties. She had undergone glossectomy due to locally advanced carcinoma which later completed five cycles of radiotherapy but further complicated with pharyngeal scarring. She had defaulted her follow up after completing her treatment.

Recently, she presented to our outpatient department with a history of recurrent cough for one year duration preceded with difficulty in swallowing for two years duration. She described the cough as chesty in nature associated with whitish sputum. It was intermittent in occurrence but later became progressively worsened as she started to have sleep disturbance due to the cough that persisted during day and night for the past three months. She also had several admissions to several private health center and finally was treated as aspiration pneumonia due to underlying swallowing difficulty. However, her condition did not improve completely. The cough was further worsened with recurrent low-grade fever, hemoptysis and weight loss of up to $7 \mathrm{~kg}$.

On further history review, her difficulty in swallowing was progressively worsened for the past one year, in which she started to have difficulty consuming even small solid foods such as rice. She only consumed a few spoons of porridge per day. She otherwise had no odynophagia, neck swelling, yellowish discoloration, abdominal pain or distension, back pain or breast mass. She admitted having choking sensation if consumed larger size of food.

Upon examination in our clinic, her height was 160 $\mathrm{cm}$, weighed $35 \mathrm{~kg}$ with body mass index (BMI) of $13.7 \mathrm{Kg} / \mathrm{M}^{2}$. She looked cachexic with prominent muscle wasting over masseter and temporalis muscles. Her lungs examination revealed the presence of consolidation of her right lower zone. Other system examinations were unremarkable.

Our investigations revealed presence of acid-fast bacilli from her sputum direct smear. Her chest Xray revealed heterogenous opacities with air bronchogram confined at right lower zone as shown in Figure 1 below.

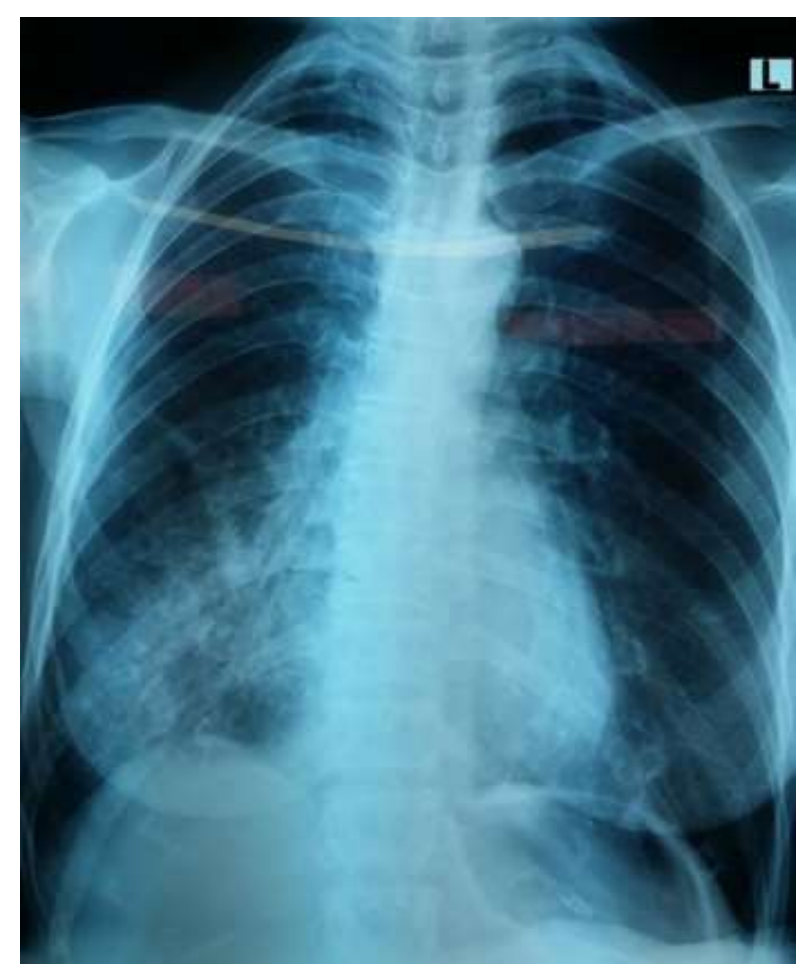

Figure 1: The chest $x$ ray of the patient in erect position and postero-anterior view with features of air-bronchogram at right lower zone. 
We treated this patient's condition as pulmonary tuberculosis with underlying malnutrition due to pharyngeal scarring secondary to post radiotherapy of her tongue carcinoma. Health district office had been notified well and contact tracing was done successfully. However, none of her family members were infected. She had been initiated with antituberculosis medications. In view of her intolerance to the large size of a single tablet of Arkurit, we have changed her medication into four individual pills of Ethambutol, Isoniazid, Rifampicin and Pyrazinamide which are relatively smaller about $1 \mathrm{~cm}$ in diameter size of each pill. Our pharmacists also educate her to crush and mix the medications with fluids for easy swallowing process. We also had referred her to otorhinolaryngology (ORL) department for swallowing test and direct laryngoscopic procedure to identify any new abnormality especially within orolaryngopharyngeal area, to rule out possibility of recurrence of her oral pharyngeal carcinoma. The shared management with the ORL team revealed that she did not have any new lesions of her oropharyngeal areas. However, she requires modification of her nutrition intake to allow the foods to be able to pass through her narrowed oral pathway.

Despite her compliance, we realized that her condition is not improved in view of her underlying underweight and poor nutrition. She also had concomitant iron deficiency anaemia. Therefore, we had involved the dietitian and her family members in our discussion to increase her weight and nutritional intake. This include adapting high caloric intake diet in a form of semisolid foods, small in amount but frequent in meals. Upon further assessment, she also had underlying moderate depressive symptoms which contributed further to her poor oral intake and defaulted follow up for her previous tongue carcinoma follow up. We were finally able to boost her motivation and self-esteem with behavior modification and antidepressant medications, which were initiated after she had completed her intensive period of antituberculosis. We also had conducted several family meetings to increase her psychosocial support and emotional care by her husband, children and parents. With multiple team approaches and management of her biological, social and spiritual problems, she was able to be managed well at our primary care center. She finally able to complete her antituberculosis treatment after eight months durations. Her weight had increased tremendously, and she was able to cope with her stress well.

\section{DISCUSSION}

The Family Medicine Specialist should be the doctor who takes professional responsibility for the health care and well-being of their patients, family members and community under territory care of the clinic. Holistic approach at the best interest of the patient should be upheld by family physicians by exploring patients' underlying biological, social, psychosocial and spiritual aspect of their disease or complaints. ${ }^{18,19}$ This includes exploring the previous illness and control, emphasizing on the understanding of the disease and morbidities, promoting healthy lifestyle and performing appropriate regular screening for their patients in order to involve in good care even at primordial level of the disease and improved patient's quality of life by preventing complications and adverse events. This case highlights a housewife who suffers chronic cough for the past one year with a background history of swallowing difficulties for several years. One of the contributing factors for the late detection in this case is the less preference of this patient to follow up at the same centre and reluctant to seek attention at nearby government clinics. This case reflects the importance of maintaining trust between the health care providers and patients in order to prevent non-compliant and defaulted treatment from recurs. ${ }^{18,19}$ Lack of continuity in care and follow up will lead to misdiagnosis of important common disease especially in relation with public safety condition. Murtagh approach in listing out the differential diagnoses even at first visit should be practiced in any centre to avoid the delayed in the diagnostic process. Therefore, the role of family physician in this case starts at developing understanding of the patient regarding the disease, thorough education and misconception correction.

Oropharyngeal carcinoma is a small adjunct of head and neck cancer. Most head and neck carcinoma is locally advanced with a high curative rate and accounts as the sixth commonest cancer in the world. ${ }^{16,20}$ Our patient had a history of tongue cancer in which she initially presented with tongue mass and pain, which was surgically treated by glossectomy and radiotherapy. As primary health care providers, our role is not only restricted at the detection process of the disease and appropriate referral, however our big responsibility also hugely involve in the postoperative period in order to help patients coping well with the disease, adapting with the swallowing process, improving the ability to speak and communicate effectively in order to achieve good quality of life despite multiple morbidities. ${ }^{15,21}$ In fact, we are also the frontliners in the detection for recurrence of the cancer. Studies have shown that late complications and morbidities usually started to occur several years after treatment of the cancer, up to $80 \%$ of cases. ${ }^{15}$ Thus, the main aims in follow up this patient include to assess clinical response and late effects of the treatment including complications; to detect recurrences; and most importantly to restore nutritional and psychosocial status of the patient. ${ }^{21}$ 
Our patient admitted that she had difficulty in swallowing and phonating after underwent the radiotherapy of her oropharyngeal area. ${ }^{13,16} \mathrm{Her}$ condition had worsened further for the past few years. She also worries about the possibility of subsequent surgery and radiotherapy, hence she avoids seeking treatment and follow up. Family physicians therefore play an important role to explore the underlying psychosocial issues surrounding the patient, especially when dealing with chronic debilitating disease and causing multiple morbidities to the patient and family members. Emotional issues and mental health should be the focus of family physicians when dealing with probable stigma that is usually related with infectious disease including pulmonary tuberculosis. Our patient also suffers depression with chronic symptoms of low mood, lack of motivation, lack of enjoyment of life, feeling worthlessness, indecisiveness, and fatigue for the past three years. This is contributed further by her difficulty in speech and inability to communicate well with her children and husband. She also felt useless and often surrounded by negative thoughts. This case highlights importance of screening for mental illness and neurotic symptoms especially in those patients with chronic diseases and cancer, which if left behind will cause poor prognosis to the outcome of the disease. In this case, her husband and children do feel abandoned when the patient changed in behaviour. Poor communication also causes the family members unable to understand the patient as a whole and help patient to relieve her pain or worries. Our patient most of the time kept her worries on her own and this burdened further her condition.

Being a manager and leader, in treating this case, family physicians should be able to delegate some important jobs among his or her supporting staff in treating the patient holistically. ${ }^{18,19}$ This includes involvement of pharmacists to give further information about the medications and converting table to liquid formulary, occupational therapist for relaxation techniques and chewing exercise and last but not least the dietician for increasing her appropriate diet intake. Antituberculosis medications are available in syrup formulary which includes for Rifampicin. However, for Isoniazid, Ethambutol and Pyrazinamide, the drugs can be crushed and mix with liquids but require correct technique and practical advice by the pharmacists. Here, a crucial role of both pharmacist and family physician is important in helping the patient. Our patient unable to tolerate an Arkurit tablet as she felt it stuck in her throat and require longer time to swallow. Our dietitian and occupational therapist also provide elaborative practical explanation for her to swallow the syrup medications effectively to avoid aspirations pneumonia from happening. This includes proper positioning while swallowing and adapt sip manoeuvre rather than gulping. Modification of her diet has also been done to provide high calorie diet. This includes eating five times meals a day consist of semisolid starchy carbohydrates with whole full-fat milk as her regular drink. Family physician also require his or her counselling skills to convince patient to start treatment and motivate patient to comply with direct observational therapy (DOTS). In this patient, with adequate information, she was able to come to our clinic for DOTS despite her initial reluctance. We also arrange some modification by allowing her husband to observe her for treatment compliance at home after two weeks DOTS at clinic for the best interest of her while maintaining the rights of public safety and avoiding transmission to the community.

Evaluation of anemia is one of the commonest problems encountered in primary care. As primary health care providers, simple and relevant investigations should be done to narrow down our diagnosis. However, in view of her acute condition and significant weight loss and swallowing difficulties, we had referred her for an urgent laryngoscope under ORL team and she was reassured to be free from any oropharyngeal carcinoma. Her hemoglobin level later was improved with oral hematinic and with reduction of pill burden after she had entered the maintenance phase of anti-tuberculosis treatment.

Family physician also serves an important role not only in providing treatment to patients but also providing various health education for the family members and community. ${ }^{18,19}$ In relation to this case, contact tracing among family members with the patient's knowledge should be done in order to protect the public safety. Ongoing talks and discussion with family members for emotional strength and psychosocial support towards patient are indeed useful. The condition of this patient had been explained well to the family members including her comorbid of depression. Her family members later able to help her well and understand her changes upon understanding the condition. This includes by adapting communicating using written communication using pen and papers. Indeed, her strong family support that she had finally received while on our treatment is one of the main key factors for her recovery from her psychosocial stress. Family physicians should be able to manage social stigma well by providing education to the community about treatment of tuberculosis and emphasize the role of community members as team members and not becoming prejudiced to patients. Family members support should be able to be brought in by a family physician by conducting meetings with them by patient's permission to understand the psychodynamic of the family and encourage them to give full support to the patient while adhering to the medications, follow up and shared care at tertiary center. This is true for our patient case in view of her underlying defaulted follow up for her 
carcinoma and long duration of symptoms. Upon discussion with the patient, one of the important reasons for her defaulted follow up of her tongue carcinoma is due to miscommunication between her and the health care providers. Lack of adequate communication will lead to misconception and wrong understanding by the patient, especially on the risk of recurrence and importance of surveillance. This case highlights the importance of good communication skills with empathetic tone using the appropriate language at the same level of patients are important.

\section{CONCLUSION}

Family physician is the leader in the health clinic. Their role is vital in giving holistic care approaches to the patient from biological, social, spiritual and psychological point of view. Education about the disease should be delivered to the patient and family members to provide better adherence to the medications and therefore better outcome in the patient. Dealing with patients with underlying history of carcinoma will require early detection of recurrence or complications and prompt action to refer to tertiary center when indicated by the family medicine specialists. This case proved that treatment of pulmonary tuberculosis in a special condition with challenging morbidities is still possible at primary care level with adequate family support and teamwork lead by the family physician.

\section{REFERENCES}

1. WHO. Cancer - Oral Cancer. World Health Organization 2020. Available at https://www.who.int/cancer/prevention /diagnosis-screening/oral-cancer/en/. Accessed January 2, 2021.

2. Kant S, Gupta H, Ahluwalia S. Significance of nutrition in pulmonary tuberculosis. Crit Rev Food Sci Nutr. 2015;55(7):955-63. doi: $\quad 10.1080 / 10408398.2012 .679500$. PMID: 24915351.

2. Lai SW, Lin CL, Liao KF. Head and neck cancer associated with increased rate of pulmonary tuberculosis in a populationbased cohort study. Medicine (Baltimore). 2017;96(43):e8366. doi:10.1097/MD.0000000000008366

4. Agyeman AA \& Ofori-Asenso. Tuberculosis - An Overview. Journal of Public Health and Emergency. 2017;1(1):1-11.

5. Pozniak A. Clinical Manifestation and Complication of Pulmonary Tuberculosis. UpToDate 2017. Available at https: / / www-uptodate-com.

/contents/clinical-manifestations-and- complications-of-pulmonary-tuberculosis. Accessed June 20, 2017.

6. Arthur T \& Evans RG. Approach to the Patient with Unintentional Weight Loss. UpToDate. 2017. Available at https://www.uptodate.com/contents/ap proach-to-the-patient-with-

unintentional-weight.

September 16, 2017.

Accessed

7. WHO. Tuberculosis - Global Tuberculosis Report 2016. World Health Organization 2016. Available at www.who.int , publications > global_report. Accessed June 20, 2017.

8. Yousang KJ, Hyun-Kyung L, Young SL \& et al. Duration of Pulmonary Tuberculosis Infectiousness under Adequate Therapy, as Assessed Using Induced Sputum Samples. Tuberculosis Respiratory Disease. 2016;80(1):27-34.

9. Zachary KC. Tuberculosis Transmission and Control. UpToDate 2017. Available at https: / /www-uptodate-

com.ezlib.iium.edu.my/contents/tuberc ulosis-transmission-and-control. Accessed September 9, 2017.

10. CDC. Center for Disease Control and Prevention: Tuberculosis CDC 2017. Available at https://www.cdc.gov/tb/default.htm. Accessed September 23, 2017.

11. Jamshed BB, Narainder G., Parveen G. \& et al. Imaging in Tuberculosis. Cold Spring Harbor Perspective in Medicine. 2015;5(6): a017814.

12. Marchiori DM. Chapter 21 - Introduction to Chest Radiography. In: Marchiori DM, ed. Clinical Imaging (Third Edition). Saint Louis: Mosby; 2014:1153-1165.

13. Larissa Nekhlyudov CS. Overview of Cancer Survivorship Care for Primary Care and Oncology Providers. UpToDate 2017. Available at https://wwwuptodate/contents/overview-of-cancersurvivorship-care-for-primary-care-andoncology-providers. Accessed June 20, 2017.

14. Schliemann D., Ismail R., Donnelly M. \& et.al. Cancer Symptom and Risk Factor Awareness in Malaysia: Findings from A Nationwide Cross-sectional Study. BMC Public Health. 2020;20(464).

15. Andrzej Kawecki RK. Follow-up In Patients Treated for Head and Neck Cancer. 
Magazine of European Medical Oncology 2014;7:87-91.

16. Bruce E, Brockstein KMS \& Shiyu S. Overview of Treatment of Head and Neck Cancer. UpToDate 2017. Available at https://www-uptodatecom.ezlib.iium.edu.my/contents/overvie W-of-treatment-for-head-and-neckcancer. Accessed June 20, 2017.

17. Reichman LB \& Lardizabal AA. Adherence to Tuberculosis Treatment. UpToDate 2020. Available at https://www.uptodate.com/contents/ad herence-to-tuberculosis-treatment. Accessed July 20, 2020.

18. Shalihin SE, Osman IF \& Din H. Assessment of Diabetic Patient Waiting Time in A Primary Healthcare Clinic. International
Medical Journal of Malaysia. 2020;19(2):41-48

19. Shalihin SE, Osman IF \& Harun Z. Non Specific Skin Purpura. Malaysian Family Physician. 2018;13(2):39-41.

20. Stenson KM. Epidemiology and Risk Factors for Head and Neck Cancer. UpToDate 2017. Available at https://www-uptodatecom.ezlib.iium.edu.my/contents/epidem iology-and-risk-factors-for-head-andneck-cancer. Accessed June 20, 2017.

21. Nerina Denaro MCM \& Elvio Grazioso R. Follow-up in Head and Neck Cancer: Do More Does It Mean Do Better? A Systematic Review and Our Proposal Based on Our Experience. Clinical and Experimental Otorhinolaryngology. 2016;9(4):287-297. 